\title{
Full computation of massive AGB evolution
}

\section{The large impact of convection on nucleosynthesis}

\author{
P. Ventura and F. D’Antona
}

\author{
Osservatorio Astronomico di Roma, via Frascati 33, 00040 MontePorzio Catone, Italy \\ e-mail: [ventura;dantona]@mporzio.astro.it
}

Received 27 August 2004 / Accepted 3 October 2004

\begin{abstract}
It is well appreciated that the description of overadiabatic convection affects the structure of the envelopes of luminous asymptotic giant branch (AGB) stars in the phase of "hot bottom burning" (HBB). We stress that this important uncertainty in the modeling plays a role which is much more dramatic than the role which can be ascribed, e.g., to the uncertainty in the nuclear cross-sections. Due to the role tentatively attributed today to the HBB nucleosynthesis as the site of self-enrichment of Globular Clusters stars, it is necessary to explore the difference in nucleosynthesis obtained by different prescriptions for convection. We present results of detailed evolutionary calculations of the evolution of stars of intermediate mass during the AGB phase for the metallicity typical of the Globular Clusters that show the largest spread in CNO abundances $\left(Z \sim 10^{-3}\right)$. We follow carefully the nucleosynthesis at the base of the external convective region, showing that very different results can be obtained according to the presciption adopted to find out the temperature gradient within the instability regions. We discuss the uncertainties in the yields of the various chemical species and the role which these sources can play as polluters of the interstellar medium.
\end{abstract}

Key words. stars: evolution - stars: interiors - stars: AGB and post-AGB - stars: abundances

\section{Introduction}

The intermediate mass stars (i.e. stars with initial masses $1 \leq$ $M / M_{\odot} \leq 7$, hereinafter IMS) experience a phase of thermal pulses (TPs) shortly after the exhaustion of central helium (Schwarzschild \& Harm 1965, 1967; Iben 1975, 1976). A CNO-burning shell supplies the global nuclear energy for $\sim 90 \%$ of the time; periodically, a thermal instability (associated with the narrow dimensions of the helium-rich layer above the $\mathrm{CO}$ degenerate core and with the steep dependence of the crosssection of $3 \alpha$ reactions on the temperature) triggers a runawaylike ignition of helium burning, which temporarily extinguishes the H-burning shell. As the mass of the CO core increases, the CNO-burning shell becomes hotter and hotter, so that the luminosity of the star increases: on the HR diagram the evolutionary tracks climb the so called "Asymptotic Giant Branch" (hence the term "AGB evolution", commonly adopted). During their evolution these structures suffer strong mass losses, which eventually stop their AGB evolution, leaving just a central remnant which later evolves into a $\mathrm{CO}$ white dwarf.

In the last decades the interest in the detailed evolution of these stars has grown, also because they have been suggested as pollutors of the medium during the early phases of evolution of globular clusters (GCs), and responsible for the chemical anomalies (in terms, e.g., of oxygen-sodium and magnesiumaluminium anticorrelations) which are observed in giants and turn-off stars of GCs. At least in the latter because of the low temperatures involved the chemical anomalies cannot be due to "in-situ" processes. The idea behind this hypothesis (to which we usually refer as "self-enrichment") is that the base of the external envelope of the most massive IMS during the AGB evolution may become so hot $\left(T_{\text {bce }}>30 \times 10^{6} \mathrm{~K}\right)$ that it triggers strong nucleosynthesis; the consequent changes in the originary chemical abundances might easily reach the surface of the star because of the rapidity of the convective motions, so that the ejecta of these stars are contaminated by such an advanced nucleosynthesis. The velocity of the ejected material should be sufficiently low to survive ejection from the cluster (D'Antona et al. 1983; Ventura et al. 2001, 2002).

The self-pollution scenario is not without its shortcomings, as pointed out by Denissenkov \& Herwig (2003): the temperatures needed to deplete oxygen would also lead to sodium destruction, and to magnesium isotopic $\operatorname{ratios}{ }^{25} \mathrm{Mg} /{ }^{24} \mathrm{Mg}$ and ${ }^{26} \mathrm{Mg} /{ }^{24} \mathrm{Mg}$ much larger than observed.

Unfortunately, the theoretical models for the AGB evolution are characterized by several uncertainties associated with the input physics which is commonly adopted in the stellar evolutionary codes: in the last decade the attention of modelists has been mainly focused on: a) the amount of extra-mixing from the base of the external region (Herwig 2000, 2004), which favours a larger efficiency of the so called third dredge-up phenomenon (i.e. the external convective region which reaches 
stellar layers previously touched by efficient $3 \alpha$ burning after a thermal pulse); b) the rate of mass loss suffered by these stars; c) the nuclear network adopted, particularly concerning the nucleosynthesis of neon, sodium and magnesium isotopes.

The present work does not discuss the plausibility of the self-enrichment scenario, but is aimed at pointing out quantitatively that, before going into the details of the chemical composition of the ejecta of AGBs, it is mandatory to understand to which extent the results obtained depend on the assumptions made in calculating the models.

We focus our attention on one of the most relevant uncertainties connected with stellar evolution, i.e. the treatment of convection, whose influence upon AGB evolution in the context of HBB is well documented in the literature, starting with the classic Renzini \& Voli (1981) paper, and with the discussion by Sackmann \& Boothroyd (1991) and Blöcker \& Schönberner (1991). Finally, D’Antona \& Mazzitelli (1996) discussed that the "Full Spectrum of Turbulence" (FST, Canuto \& Mazzitelli 1991) model for convection leads to a much more efficient HBB than the Mixing Length Theory (MLT). Neverthless, in these last years, no mention has been made in many recent detailed computations of the description adopted for convection, and, more importantly, of the resulting uncertainty in the nucleosynthesis.

We will compare the results obtained with the two local models currently available, i.e. MLT and the FST convective model; within the MLT framework, we discuss the role played by the free parameter $\alpha$.

We show that the main physical properties of the models depend strongly on the efficiency of convection, and discuss the implications for the self-enrichment scenario.

\section{The evolutionary code}

The stellar evolution models discussed in this paper were calculated by the code ATON, a full description of which can be found in Ventura et al. (1998) (ATON2.0 version). In this paper the interested reader may find a detailed description of the numerical structure of the code, and of the macro- and microphysics used to simulate the stellar evolutions.

The code has now been updated concerning the nuclear network, which has been widened in order to include more chemical elements and nuclear reactions, according to the detailed description given below. The current version is therefore ATON2.1.

Here we briefly recall the most important input data adopted.

\subsection{The convective model}

The code allows us to calculate the temperature gradient within instability regions either by adopting the traditional MLT (Vitense 1953; Böhm-Vitense 1958), or the FST model (Canuto \& Mazzitelli 1991; Canuto et al. 1996). The interested reader may find a detailed description of the physical differences between the two models in Canuto \& Mazzitelli (1991).

Briefly, we recall that within the MLT scheme both the dimensions of the convective eddies and the mixing length are assumed to be directly proportional to the local value of $H_{\mathrm{p}}$, the pressure scale height $\left(l=\alpha H_{\mathrm{p}}\right)$; the free parameter $\alpha$ is calculated in order to reproduce the evolution of the Sun. The most recent estimates give $\alpha=1.7$. In the FST model the mean dimension of the convective eddies is found by integrating over the whole spectrum of the dimensions, and the mixing length is simply assumed to be $l=z$, the distance from the closest convective boundary.

The differences between the results provided by the two models can be summarized as follows:

- within high-efficiency convective regions (i.e. stellar regions where most of the energy is carried by convection) the level of overadiabaticity $\nabla-\nabla_{\mathrm{ad}}$, (where $\frac{\nabla=\mathrm{d} \log T}{\mathrm{~d} \log P}$ ) required by the FST models is lower;

- within low-efficiency instability regions (e.g. low density convective zones) the FST model exhibits a superadiabaticity peak which is not found within the MLT framework.

\subsection{Nuclear network}

With respect to Ventura et al. (1998) the nuclear network has been widened and now includes 30 chemicals: $\mathrm{H}, \mathrm{D}$, ${ }^{3} \mathrm{He},{ }^{4} \mathrm{He},{ }^{7} \mathrm{Be},{ }^{7} \mathrm{Li},{ }^{12} \mathrm{C},{ }^{13} \mathrm{C},{ }^{14} \mathrm{~N},{ }^{15} \mathrm{~N},{ }^{16} \mathrm{O},{ }^{17} \mathrm{O},{ }^{18} \mathrm{O},{ }^{18} \mathrm{~F},{ }^{19} \mathrm{~F},{ }^{20} \mathrm{Ne}$, ${ }^{21} \mathrm{Ne},{ }^{22} \mathrm{Ne},{ }^{22} \mathrm{Na},{ }^{23} \mathrm{Na},{ }^{24} \mathrm{Mg},{ }^{25} \mathrm{Mg},{ }^{26} \mathrm{Mg},{ }^{26} \mathrm{Al},{ }^{27} \mathrm{Al},{ }^{28} \mathrm{Si},{ }^{29} \mathrm{Si}$, ${ }^{30} \mathrm{Si},{ }^{31} \mathrm{P}, \mathrm{n}$. The nuclear reactions considered are 64 . They include all the main reactions of the p-p, CNO, Ne-Na and $\mathrm{Mg}$ Al chains, and the $\alpha$ captures of all nuclei up to ${ }^{26} \mathrm{Mg}$. The relevant cross-sections can be taken either from Caughlan \& Fowler (1988) or from Angulo et al. (1999).

\subsection{Convective mixing and nuclear burning}

Since we expect a non-negligible fraction of the global nuclear release to be generated within the convectively unstable external regions, we decided to adopt for all the evolution models presented here a diffusive algorithm to deal with nuclear burning within convective regions, in which nuclear burning and mixing of chemicals are coupled self-consistently. We therefore solve for each element the diffusion equation (Cloutman \& Eoll 1976):

$\left(\frac{\mathrm{d} X_{i}}{\mathrm{~d} t}\right)=\left(\frac{\partial X_{i}}{\partial t}\right)_{\text {nucl }}+\frac{\partial}{\partial m_{r}}\left[\left(4 \pi r^{2} \rho\right)^{2} D \frac{\partial X_{i}}{\partial m_{r}}\right]$

stating mass conservation of element $i$. The diffusion coefficient $D$ is taken as

$D=\frac{1}{3} u l$

where $u$ is the convective velocity and $l$ is the convective scale length.

Within this diffusive framework it is necessary to specify the way with which convective velocities decay outside the convective boundaries (Deng at al. 1996a,b; Herwig et al. 1997; Ventura et al. 1998). In agreement with Xiong (1985) and Grossman (1996) and supported by the numerical simulations 
by Freytag et al. (1996), we assume that convective velocities decay exponentially outside the formal convective boundary as:

$u=u_{\mathrm{b}} \exp \pm\left(\frac{1}{\zeta f_{\text {thick }}} \ln \frac{P}{P_{\mathrm{b}}}\right)$

where $u_{\mathrm{b}}$ and $P_{\mathrm{b}}$ are, respectively, turbulent velocity and pressure at the convective boundary, $P$ is the local pressure, $\zeta$ a free parameter connected with the e-folding distance of the decay, and $f_{\text {thick }}$ is the thickness of the convective regions in fractions of $H_{\mathrm{p}}$.

\section{Early evolutionary phases}

We computed three evolutions starting with initial masses $M=$ $5 M_{\odot}$. With the exception of the convective model, all the physical and chemical input data adopted are the same, i.e.:

- Since our purpose is to test how the uncertainties connected with convection may reflect on the plausibility of the "selfenrichment" scenario, we adopt a chemistry which is typical of those GCs which show most of self-enrichment like M 13, NGC 6752 (Gratton et al. 2001; Sneden et al. 2004), i.e. $Z=0.001$ and $Y=0.24$. For all chemicals other than helium we adopted solar-scaled abundances.

- We assumed extra mixing from the external border of any convective region as given by a parameter $\zeta=0.02$ for the exponential decay of velocities starting from the formal convective border; this is in agreement with the calibration given in Ventura et al. (1998). Since our purpose is to discuss the uncertainties connected with the treatment of convection, no inwards overshooting from the base of the external convective region was adopted.

- For mass loss, Blöcker's (1995) prescription was used in all cases: the free parameter $\eta_{R}$ entering Blöcker's formula was set to $\eta_{R}=0.02$, in agreement with the calibration for mass loss during the AGB evolution given in Ventura et al. (2000).

- The Angulo et al. (1999) cross-sections were used.

- Two models were calculated with an MLT treatment of convection with parameters $\alpha=1.7$ (MLT17 model) and $\alpha=2.1$ (MLT21 model). We also calculated an evolution model with the FST convective prescription (FST model).

Figure 1 shows the tracks in the HR diagram corresponding to the three evolution models discussed above. We note that the tracks are very similar during the two major phases of nuclear burning within the central regions, because convection within burning cores is so efficient that the gradient is practically adiabatic, independently of the convective model. The only differences can be noticed in the colors of the red giant branch (RGB) and the early AGB evolution. These are the regions of the HR diagram where the stars develop a very extended convective envelope, in which a great part of the energy is carried by radiation because of the low convective efficiency: in these cases the temperature gradient from the CNO-burning shell to the surface (hence the effective temperature of the star) depends sensibly on the convective model adopted. A full discussion of the

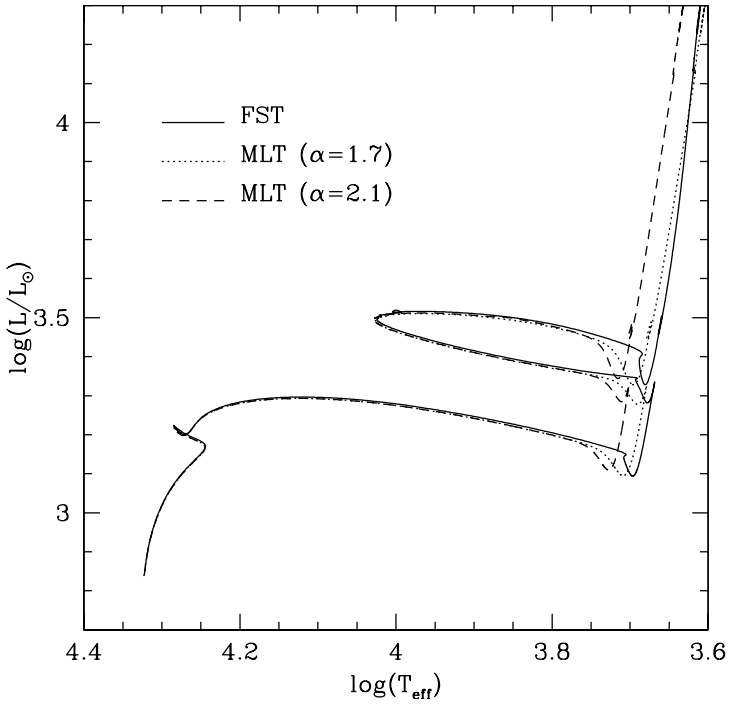

Fig. 1. The tracks in the HR diagram of three models with initial mass $5 M_{\odot}$ calculated with three different tretaments of convection. Full: FST models; dotted: MLT model with $\alpha=1.7$; dashed: MLT model with $\alpha=2.1$.

dependency on the convective model of the effective temperature of IMS during various evolutionary phases can be found in Ventura \& Castellani (2005).

What is most interesting here is that the main physical properties and the duration of the various evolutionary phases are approximately the same for the three models, because in any case the nuclear sources are either well within regions of radiative stability, or inside central cores where the convective efficiency is extremely high, making the gradient almost adiabatic.

The phase of H-burning lasts $90 \mathrm{Myr}$; during it the star develops a central convective core of $\sim 1.4 M_{\odot}$, which progressively shrinks in mass until $\mathrm{H}$-burning is extinguished in the central regions. Soon after hydrogen exhaustion the star expands, and surface convection reaches inner layers which were previously at least partly touched by nuclear burning (first dredge-up). The lowest point (in mass) reached by the base of the external envelope is for all the three models $\sim 2.1 M_{\odot}$ away from the centre; the whole duration of this phase is $\sim 70000 \mathrm{yr}$.

Shortly afterwards the central core becomes hot enough to ignite $3 \alpha$ reactions. During helium burning the star develops a central convective core of $\sim 0.5 M_{\odot}$; the total duration of this phase for the three models discussed is $\sim 12 \mathrm{Myr}$.

After the central helium exhaustion, $3 \alpha$ reactions operate in an intermediate layer above the core of carbon and oxygen. All the stellar layers above this He-burning shell expand, and the CNO-burning shell is temporarily extinguished. Convection penetrates inwards, reaching a layer which is $0.9 M_{\odot}$ away from the centre (second dredge-up). The total duration of this phase of inner penetration of surface convection is $0.55 \mathrm{Myr}$, after which the CNO-burning shell is activated again, and the star begins the AGB evolution. 


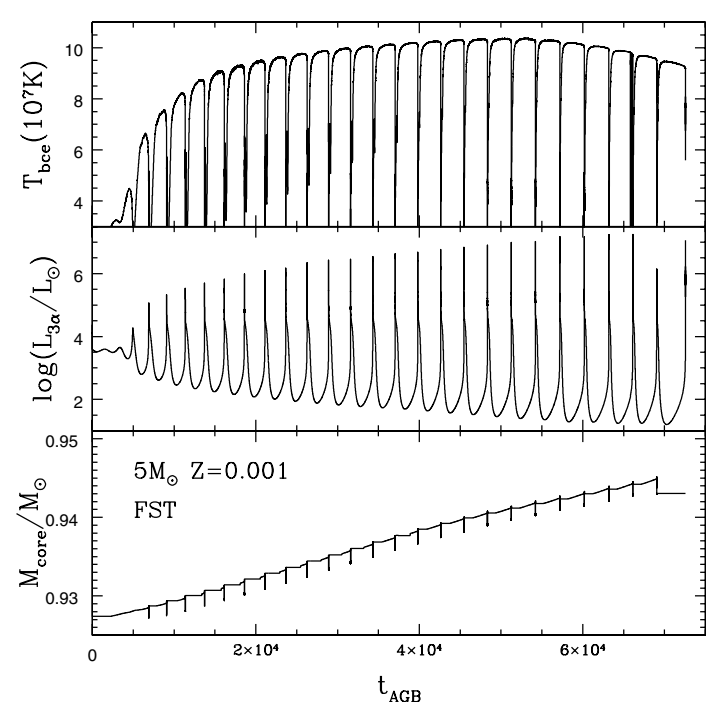

Fig. 2. The variation with time of the temperature at the base of the convective envelope (top), luminosity due to helium burning (middle), and core mass (bottom) of a $5 M_{\odot}$ FST model during the AGB evolution. Time was counted from the beginning of the AGB evolution.

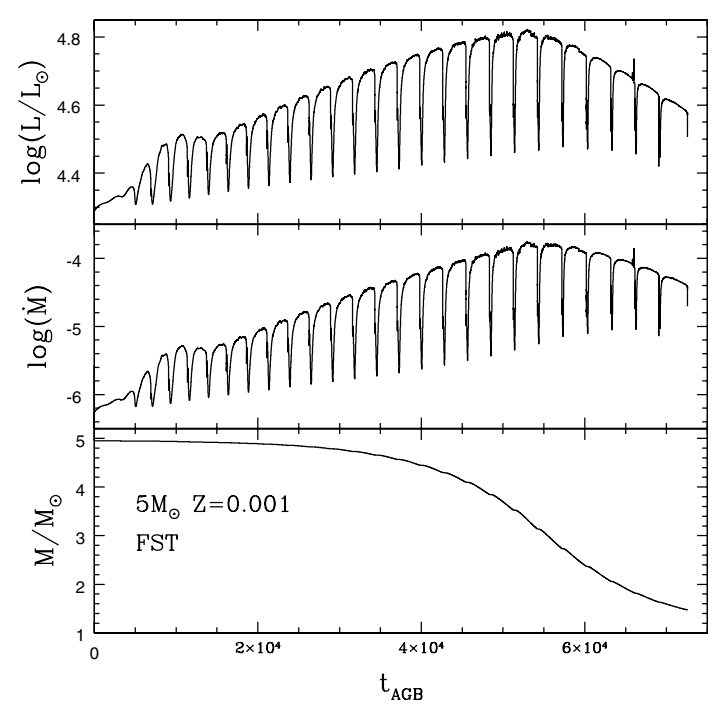

Fig. 3. The variation with time of luminosity (top), mass loss rate (middle) and total mass (bottom) of the model presented in Fig. 2.

\section{Standard AGB evolution}

Shortly after the second dredge-up the external layers of the star contract and heat, thus favouring the re-ignition of the CNO-burning shell. Hereinafter, the CNO cycle will be the only nuclear source supporting the star, with the only exception of the contribution of a $3 \alpha$ burning shell which once every $\sim 3000 \mathrm{yr}$ is ignited in a thermally unstable way, leading to a thermal pulse (TP).

Figures 2-4 show the temporal evolution of some physical quantities related to the evolution of the FST model with initial mass $5 M_{\odot}$. We note a rapid increase of the stellar luminosity (Fig. 3, top panel), which is associated with the fast increase of $T_{\text {bce }}$ (Fig. 2, top panel), which is almost doubled after three TPs. We will see that the rapidity of this rise is strongly dependent on the convective model adopted.

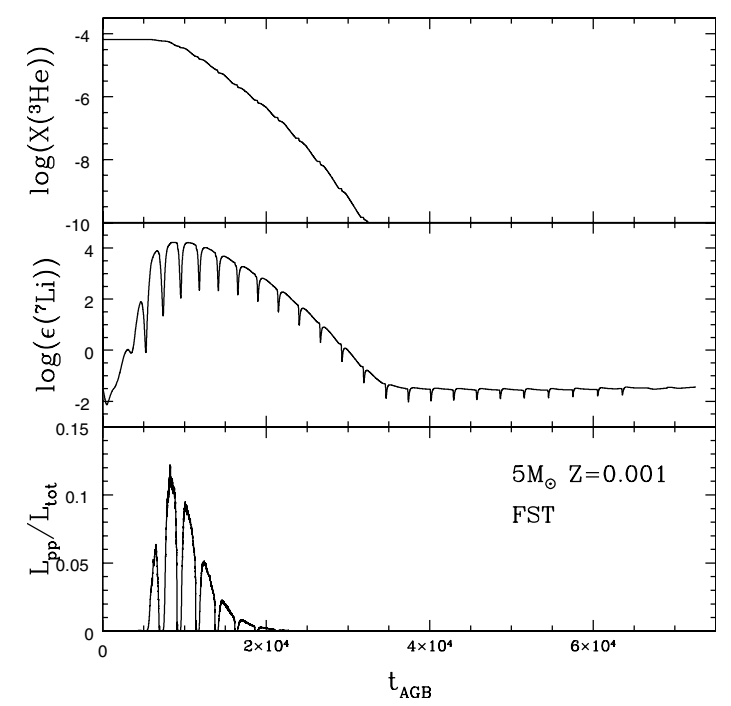

Fig. 4. Evolution with time of surface ${ }^{3} \mathrm{He}$ (top), lithium (middle) and fraction of nuclear energy due to proton-proton reactions (bottom) of the FST model presented in Fig. 2.

The periodic strong drop of the global luminosity and of $T_{\text {bce }}$ marks the ignition of TPs, which trigger an expansion of the layers above the helium burning shell with the consequent stop of CNO burning and shrinking of the surface convective envelope.

The CNO luminosity is generated in a shell which is $\delta M \sim$ $10^{-4} M_{\odot}$ wide, and which progressively moves outwards, burning at higher and higher gravities and temperatures: this determines the increase of luminosity shown in the upper panel of Fig. 3, which is eventually halted by the strong mass loss, which reduces the mass of the envelope, as can be seen in the bottom panel of Fig. 3. In the same figure we see the rapid increase of the mass loss with luminosity (middle panel): within Blöcker (1995) prescription, and with the adopted value of the free parameter $\eta_{R}$, we see that a maximum value of $\sim 2 \times 10^{-4} M_{\odot} / \mathrm{yr}$ is attained, almost in conjunction with the maximum luminosity of the star. Therefore, the rapidity with which the luminosity increases plays a fundamental role in determining the total duration of the whole AGB evolution, triggering a fast reduction of the mass of the envelope. For the model discussed here, we see that the AGB phase lasts $\sim 70000 \mathrm{yr}$.

For a few TPs we note from the bottom panel of Fig. 4 that a non-negligible fraction of the nuclear energy release is due to the proton-proton ( $p-p)$ chain: during the 4th interpulse phase this latter channel provides $\sim 10 \%$ of the luminosity of the star. This is due essentially to ${ }^{3} \mathrm{He}$ burning at the base of the external envelope (see the top panel of Fig. 4), which becomes efficient as soon as the temperature reaches $\sim 4 \times 10^{7} \mathrm{~K}$. The $\mathrm{p}-\mathrm{p}$ contribution becomes negligible as soon as the surface ${ }^{3} \mathrm{He}$ is all burnt (bottom panel). We also note that this is associated with the lithium production via the Cameron \& Fowler (1971) mechanism, as can be seen by the large increase of the surface lithium abundance shown in the middle panel of the same figure. The end of ${ }^{3} \mathrm{He}$ burning at the base of the convective envelope is associated with the temporary plateau in the 


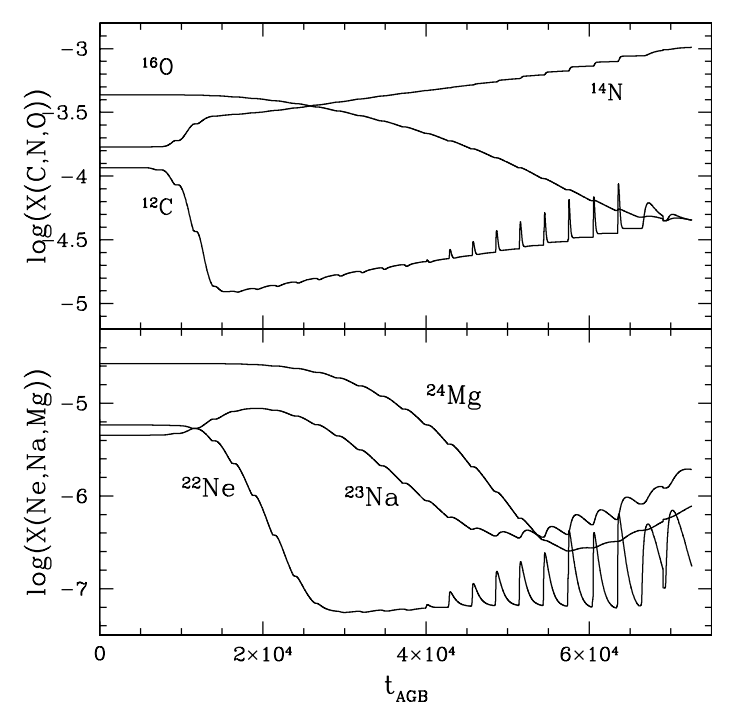

Fig. 5. Evolution with time of the surface chemical abundances of the CNO elements (top) and of some neon, sodium and magnesium isotopes (bottom) of the FST model presented in Fig. 2.

global luminosity rising which can be detected in the top panel of Fig. 3, after $\sim 12000 \mathrm{yr}$.

We end this general description of AGB evolution with a glance at the nucleosynthesis at the base of the convective envelope: we recall that convection is generally extremely fast in homogenizing the whole external region; therefore the ejecta of these stars are practically determined by the changes of the chemical abundances at the base of the external zone.

We note from the top panel of Fig. 5 that for the first $\sim 15000$ yr the oxygen abundance is unchanged, while ${ }^{14} \mathrm{~N}$ is produced at the expenses of ${ }^{12} \mathrm{C}$ : this is the signature of a $\mathrm{CN}$ cycle, which is operating at temperatures not exceeding $\sim 7 \times 10^{7} \mathrm{~K}$. Later on, when $T_{\text {bce }}$ exceeds $\sim 8 \times 10^{7} \mathrm{~K}$, oxygen is depleted at the base of the envelope, with the consequent increase of the ${ }^{14} \mathrm{~N}$ and ${ }^{12} \mathrm{C}$ abundances. After $\sim 15 \mathrm{TPs}$, we can cleary see the signature of the Third Dredge-Up (TDU) in the strong increase of the surface ${ }^{12} \mathrm{C}$ abundance following each TP. More precisely, we find the first TDU episode after 13 TPs; the efficiency parameter $\lambda$ (defined as the ratio between the mass which is dredged up following each TP and the mass gained by the $\mathrm{H}$-exhausted region since the precedent $\mathrm{TP})$ reaches an asymptotic value of $\lambda \sim 0.5$ after 19 TPs.

These results confirm, at least within the FST framework, the possibility of attaining deep oxygen burning at the base of the external convective zone of these stars: the final ${ }^{16} \mathrm{O}$ abundance is $>10$ times lower, while the ejecta would have an average oxygen content a factor of $\sim 3$ lower than the initial abundance.

From the bottom panel of Fig. 5 we see an early phase of ${ }^{22} \mathrm{Ne}$ destruction, which favours a temporary production of sodium, increased by another factor of $\sim 2$ (in the comparison of the current sodium abundance with the initial value, we must recall that the second dredge-up had already favoured a sodium increase of a factor $\sim 3$ ). Shortly after the beginning of oxygen depletion, the surface sodium abundance starts decreasing due to the NeNa chain.

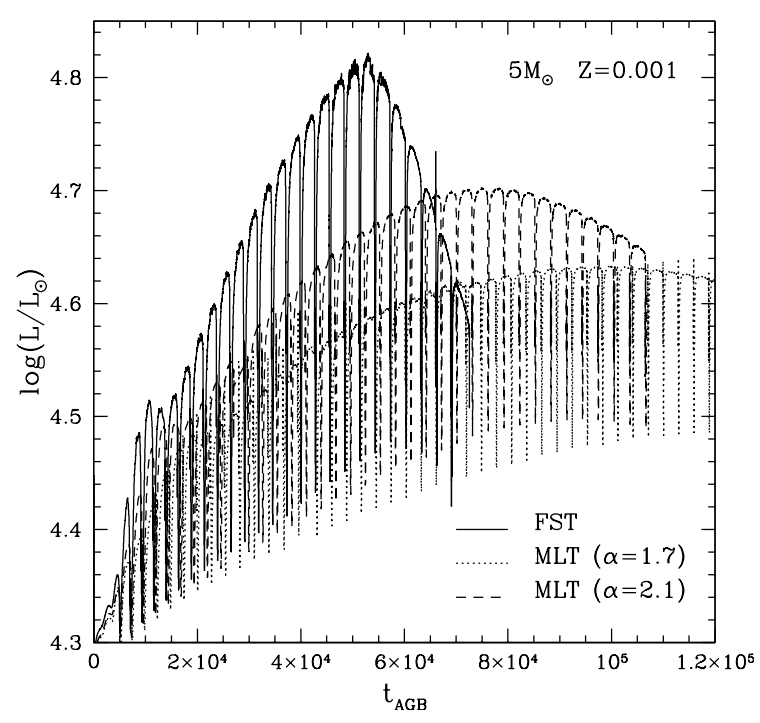

Fig. 6. The comparison of the temporal variation of the luminosity of three models with initial mass $M=5 M_{\odot}$ calculated with three different prescriptions for the treatment of the regions unstable to convection.

Later on, when TDU becomes operative, a considerable amount of ${ }^{22} \mathrm{Ne}$ is carried outwards to the surface following each TP: this favours sodium production again, as we see in the bottom panel of Fig. 5. The overall sodium abundance of the ejecta would be sligtly lower than the initial value.

Finally, we note that ${ }^{24} \mathrm{Mg}$ is also depleted at the base of the external convective zone: the final abundance is lower with respect to the initial value by a factor of $\sim 20$.

\section{The influence of the convective model}

We stressed in Sect. 3 that prior to the TPs phase the three evolutions are very similar. Here we focus our attention on the AGB phase, keeping in mind that at the beginning of this phase the structures of the three models are practically identical.

In Fig. 6 we show the variation with time of the luminosity for the three models discussed. We see a large difference starting already at the first TPs; the more efficient the convective model is, the more rapid is the increase of luminosity as the evolution proceeds. We see that the FST model achieves a maximum luminosity which is $\sim 70 \%$ larger than the corresponding value of the MLT17 model.

This behaviour can be seen as an extreme case of the break-down of the core mass vs. luminosity relation which occurs when the hydrogen burning region is not detached from the convective region (Tuchman et al. 1983; Blöcker \& Schönberner 1991).

Such a large difference in the evolution of luminosity is inevitably reflected in the mass loss rate: we see from Fig. 7 a much faster consumption for the FST model, which loses the whole envelope within $\sim 70000 \mathrm{yr}$, to be compared to the $\sim 130000 \mathrm{yr}$ associated to the MLT21 model, and $\sim 200000 \mathrm{yr}$ of the MLT17 model. We therefore can see that the duration of the whole AGB phase may differ by a factor of $\sim 3$ according to the convective model adopted. 


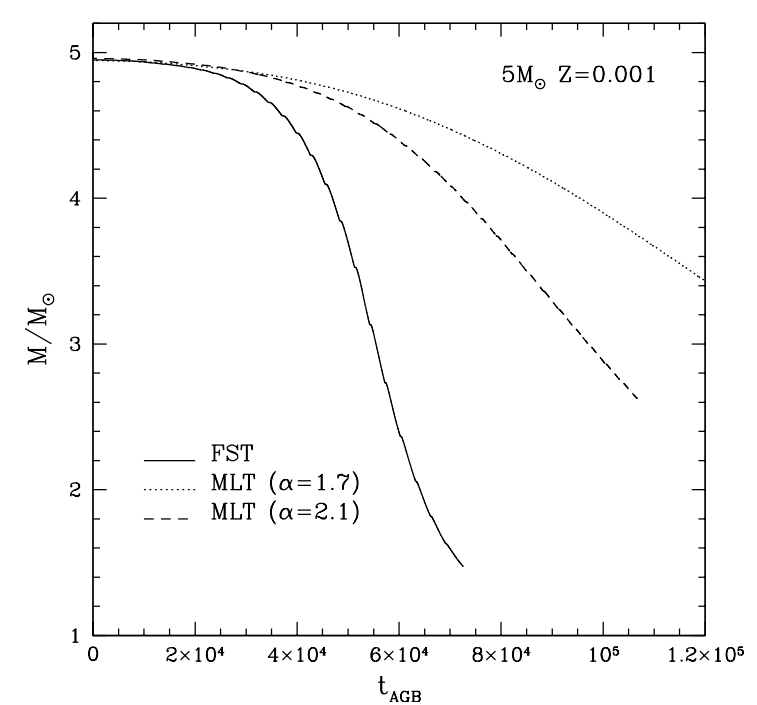

Fig. 7. The variation with time of the total mass of the models shown in Fig. 6.

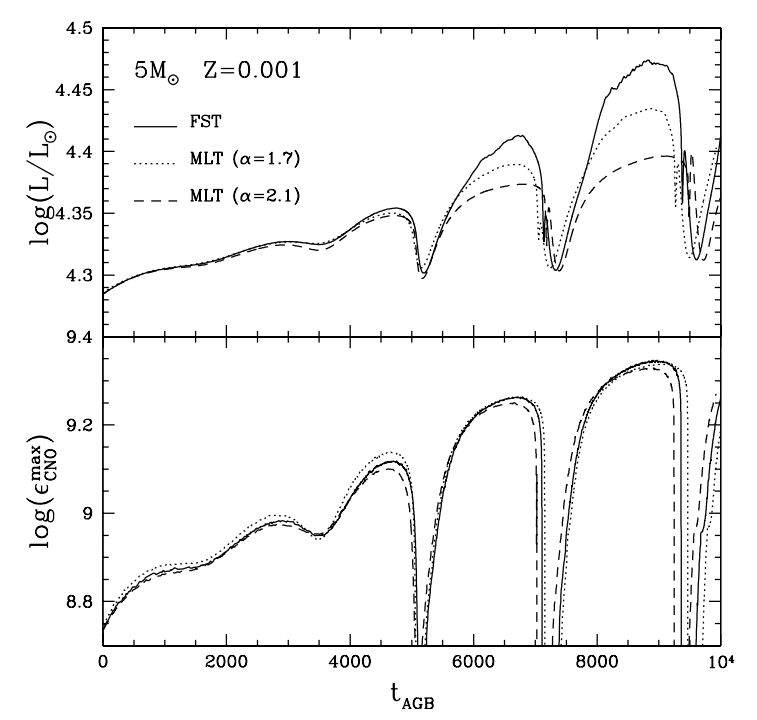

Fig. 8. The variation with time of the total luminosity (top) and of the maximum CNO nuclear energy release (bottom) for the three models discussed in Fig. 6. Only the early evolution along the AGB is shown.

Before entering into the details of the consequences which these differences may have for the chemistry of the ejecta, hence for the way in which these sources pollute the interstellar medium, we examine the reasons for such large differences.

We show in Fig. 8 the comparison between the evolution of the three models, limited to the first $10000 \mathrm{yr}$ of AGB, or, equivalently, the first 3-4 TPs. The upper panel shows the total luminosity of the star, in the bottom panel we report the maximum value of the CNO-burning coefficient for nuclear energy release. While the latter quantity is very similar for each of the three models, the luminosities diverge. We may therefore conclude that while the internal structure of the models in terms of thermal stratification and chemical profile is similar up to the CNO-burning shell, some difference must arise in the immediate proximity of the latter, close to the inner boundary of the convective envelope.

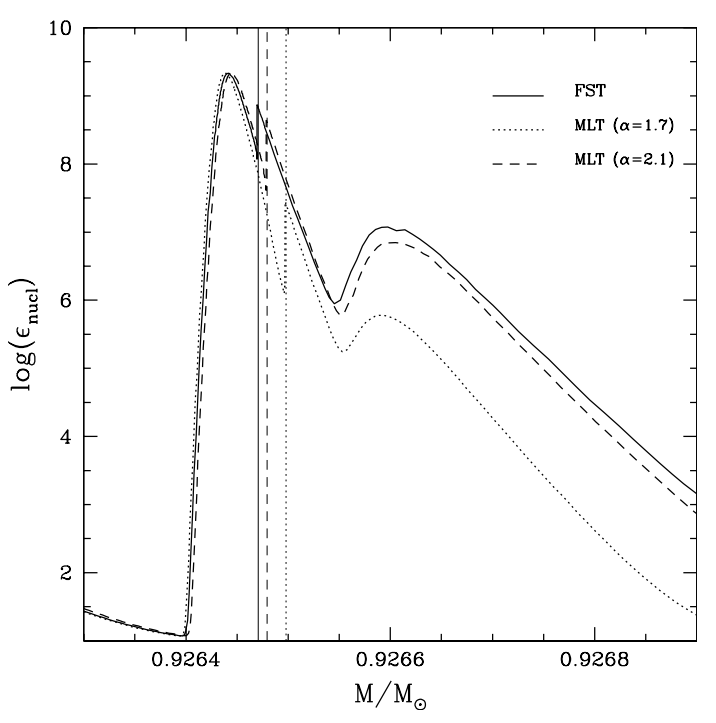

Fig. 9. The internal nuclear structure of the models presented in Fig. 6 at the maximum luminosity between the $3 \mathrm{rd}$ and 4 th TPs. The abscissa gives the distance (in solar masses) from the centre of the star, and the ordinate shows the coefficient for nuclear energy release. The thin vertical lines indicate the location of the inner border of the convective envelope.

To better clarify this point, we show in Fig. 9 the interior stratifications of the coefficient for the nuclear energy release; for all the three models the figure refers to the point of maximum luminosity during the fourth interpulse phase, $\sim 9000 \mathrm{yr}$ after the beginning of the AGB evolution. The mass coordinate of the MLT17 and MLT21 models has been artificially shifted in order to have the peak value of $\epsilon_{\text {nucl }}$ at the same abscissa. This shift is much less than $10^{-3} M_{\odot}$. The thin vertical lines indicate the location of the bottom of the convective envelope for each model.

For each of the three models we see a very similar behaviour. In the proximity of the $\epsilon_{\text {nucl }}$ peak there is a secondary maximum at the border of the surface convection, which carries ${ }^{3} \mathrm{He}$-rich material into the $\mathrm{CNO}$-burning shell; during these first TPs, as already discussed in Sect.4, the p-p contribution is non-negligible (see the bottom panel of Fig. 4). Also, we see a secondary maximum $\sim 2 \times 10^{-4} M_{\odot}$ away, which is entirely due to lithium burning; the contribution of the latter to the overall energy release, however, is negligible.

If we compare the three models, we note a strong similarity up to the peak of the shell and just beyond, with the only difference that the location of the inner border of the convective external zone is closer to the CNO peak within the FST model, while it is a few $10^{-5} M_{\odot}$ further away in the MLT17 case; the MLT2 1 model is intermediate. The proximity of the convective border to the $\mathrm{CNO}$ peak carries more ${ }^{3} \mathrm{He}$ within a region whose temperature is typical of CNO-burning regions $\left(T \sim 7 \times 10^{7} \mathrm{~K}\right)$, thus triggering a considerable extra luminosity. This extra contribution is smaller the more distant the CNO peak and the base of the envelope are, and it is practically negligible within the MLT17 model.

From the above discussion it becomes evident that in understanding the different luminosities of the models, despite 

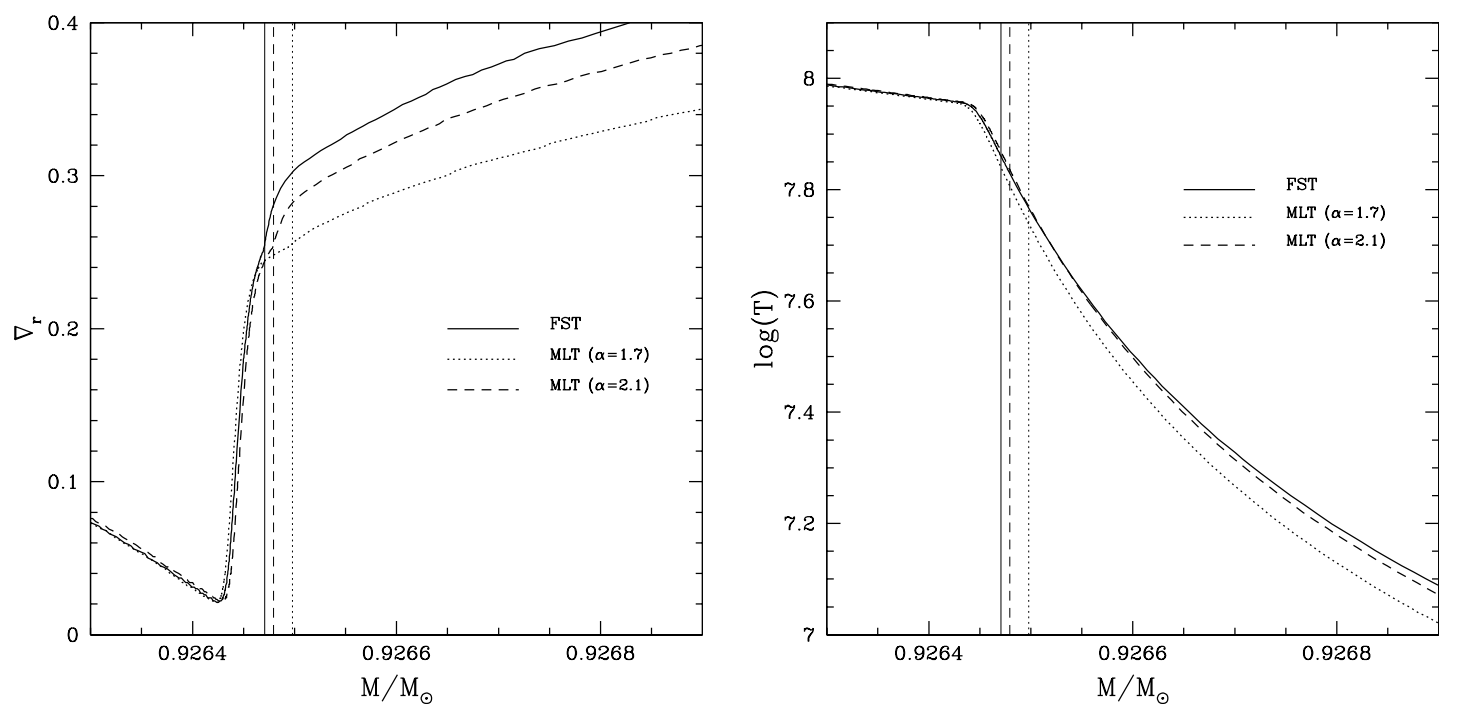

Fig. 10. Internal distribution of radiative gradient (left), and temperature (right) for the same models shown in Fig. 9.

the similarity characterizing their structures up to the CNOburning shell, a key role is played by the exact location of the inner border of the convective envelope, or, equivalently, its distance from the peak of the CNO shell. Within the framework of the Schwartzschild's criterion, which is used to fix the neutrality point according to the condition $\nabla_{\mathrm{rad}}=\nabla_{\mathrm{ad}}$, the location of the border is furhter inward the steeper the $\nabla_{\text {rad }}$ profile towards the external layers.

The comparison of the variations of the $\nabla_{\text {rad }}$ within the three models presented in Fig. 9 is reported in the left panel of Fig. 10. We see in all cases that $\nabla_{\text {rad }}$ increases from the peak of the shell, because of the rapid decrease of temperature and pressure and the increase of the opacity; near the convective region the FST $\nabla_{\text {rad }}$ profile keeps the same slope, while the MLT models, particularly MLT17, become progressively flatter, thus moving the neutral point, where $\nabla_{\mathrm{rad}}=\nabla_{\mathrm{ad}}$, outwards.

The reason for this behaviour can be found in the difference in the efficiency of the three convective models, and the relative effect on the temperature profile. It is well known that the FST model is more efficient in high-efficiency convective regions, thus requiring a lower degree of overadiabaticity to carry the same amount of energy flux by convection: in the layers next to the inner border of surface convection shown in Fig. 9 the FST overadiabaticity is $\sim 0.8$ dex smaller. In a small region very close to the border the trend is reversed, because the MLT models attain an extremely low level of overadiabaticy: this is due to a physical inconsistency of the MLT assumption that the mixing length is proportional to $H_{\mathrm{p}}$, which is meaningless near the border, where the same quantity is expected to vanish, as is consistently described in the FST ${ }^{1}$.

Within the instability regions, the fraction of energy which is carried by radiation decreases more rapidly in the FST than in the MLT models; the necessity of keeping the radiative flux $F_{\text {rad }}$ higher requires a lower and flatter profile of $\nabla_{\text {rad }}$ (we recall that $\left.F_{\text {rad }} \propto 1 / \nabla_{\text {rad }}\right)$; it is the necessity of matching this profile within

\footnotetext{
1 yet this region is so efficient convectively that this has no consequences for the thermodynamical structure of these regions, the gradient being very close to the adiabatic value.
}

the convective region which forces the MLT radiative gradient profile to bend near the border, thus shifting the location of the neutrality point outwards.

The higher efficiency of the FST model thus has two important consequences:

- The FST $\nabla_{\text {rad }}$ profile is steeper, favouring a more internal location of the formal border; this in turn favours a larger luminosity, because we have an efficient extra contribution of the $\mathrm{p}-\mathrm{p}$ chain.

- The lower value of the overadiabaticity required in the FST model (and, partially, in the MLT21 model) leads to a smaller temperature gradient within the instability region (see the right panel of Fig. 10), which, in turn, acts in favour of a larger contribution of the extra luminosity supplied by ${ }^{3} \mathrm{He}$ burning within these stellar layers.

From a chemical point of view the differences among the three models are also relevant. Figure 11 shows the variation of the abundances of some key elements included in our nuclear network; in the left panel we report the evolution of the CNO surface abundances, in the right ${ }^{20} \mathrm{Ne},{ }^{23} \mathrm{Na}$ and ${ }^{24} \mathrm{Mg}$ are shown. In this case, to have an idea of the mean chemical composition of the ejecta of the star, we use the mass as abscissa instead of time (Ventura et al. 2001); the difference is relevant, because most of the mass is lost in correspondence of the maximum luminosity, in a time interval which is very short.

We note in the left panel of Fig. 11 that all three models achieve oxygen depletion, though in MLT17 the final depletion is just by a factor of $\sim 2$. The MLT17 model, which yields a longer lifetime, experiences more TDUs, thus it produces more ${ }^{12} \mathrm{C}$ and ${ }^{14} \mathrm{~N}$. As in the FST case, we find that the parameter connected to the efficiency of the TDU approaches an asymptotic value of $\lambda \sim 0.5$ after $\sim 20$ TPs.

In the right panel we note the different behaviour of sodium. In all three cases we have an early phase of production (which is due to the combined effects of the second dredge-up and to the destruction of ${ }^{22} \mathrm{Ne}$ at the beginning of the AGB evolution), and a later phase of depletion; in the MLT models, however, 

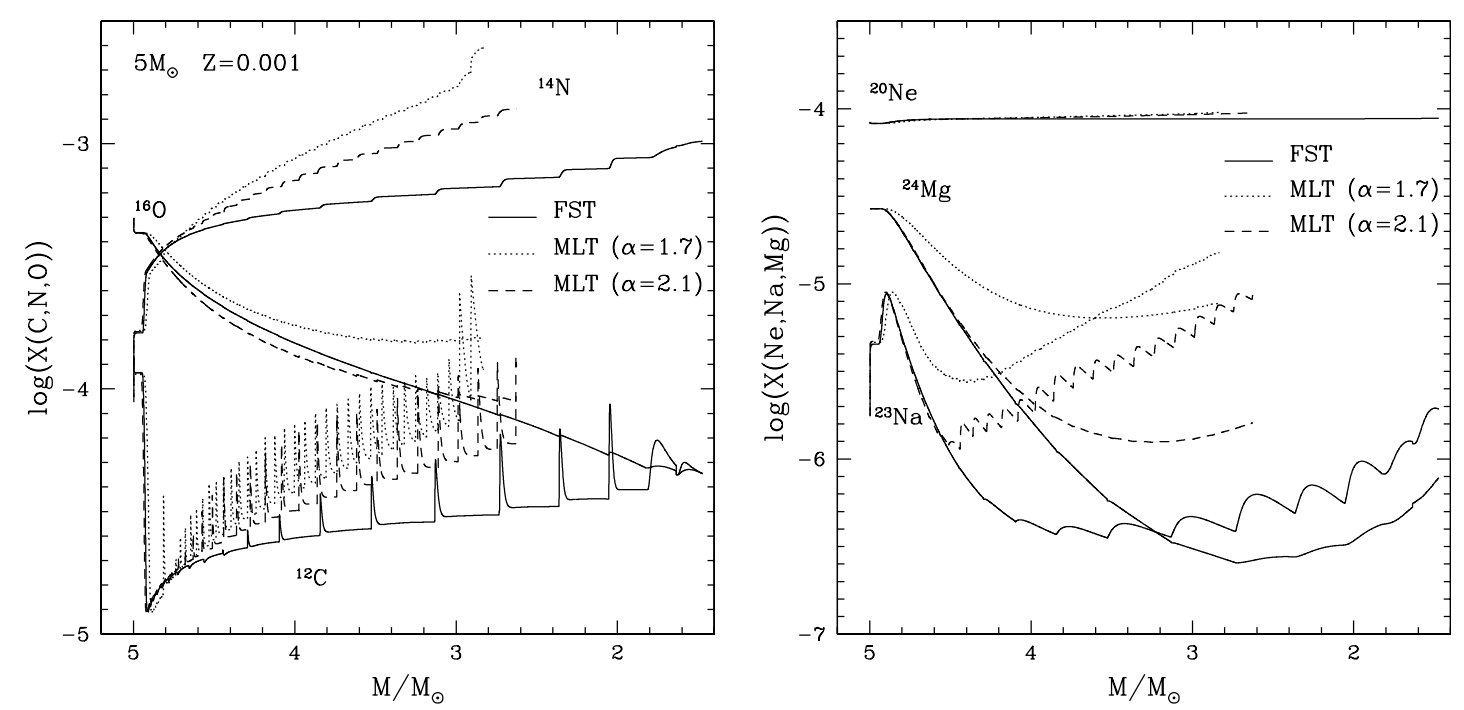

Fig. 11. The variation of the abundances of some chemicals at the surface of the star as a function of the total mass (decreasing due to mass loss). Left: CNO abundances; Right: neon, sodium and magnesium abundances.

Table 1. Chemistry of the ejecta.

\begin{tabular}{cccccccc}
\hline \hline Model & $Y^{a}$ & ${ }^{7} \mathrm{Li}^{b}$ & {$\left[{ }^{12} \mathrm{C}\right]^{c}$} & {$\left[{ }^{14} \mathrm{~N}\right]^{c}$} & {$\left[{ }^{16} \mathrm{O}\right]^{c}$} & {$\left[{ }^{23} \mathrm{Na}\right]^{c}$} & $\left.{ }^{24} \mathrm{Mg}\right]^{c}$ \\
\hline MLT17 & 0.32 & 2.0 & -0.3 & 1.51 & -0.40 & 0.78 & -0.45 \\
MLT21 & 0.32 & 1.9 & -0.5 & 1.34 & -0.55 & 0.60 & -0.83 \\
$F S T$ & 0.31 & 1.9 & -0.7 & 1.13 & -0.60 & -0.16 & -0.95 \\
\hline
\end{tabular}

a Helium mass fraction of the ejecta.

${ }^{b}$ Lithium content of the ejecta on the scale $\log \left(N\left({ }^{7} \mathrm{Li}\right)\right)=$ $\log \left({ }^{7} \mathrm{Li} / \mathrm{H}\right)+12.00$.

${ }^{c}$ Average abundances with respect to the initial value: $[X]=$ $\log \left(X_{\text {ejecta }} / X_{\text {initial }}\right)$.

the effect of several TDUs make the surface content of sodium rise again, and reach extraordinarily large values, particularly within the MLT17 model. We also note a different degree of ${ }^{24} \mathrm{Mg}$ depletion in the three cases, ${ }^{24} \mathrm{Mg}$ reduction being larger the larger the efficiency of the convective model.

Table 1 summarises the chemical composition of the ejecta for each of the three models discussed. As we may see there are just two robust conclusions which we can draw, independently of the convective model adopted:

- The fraction (in mass) of helium in the expelled material is $Y \sim 0.31$ in all cases: this can be understood on the basis of the fact that it occurs essentially during the second dredge-up, while the following production during AGB is negligible.

- The lithium content of the ejecta is $\log \left(\epsilon\left({ }^{7} \mathrm{Li}\right)\right) \sim 1.9$, slightly less than the population II average abundance. This is because the phases of lithium production and destruction happen at the very beginning of the AGB evolution, when the differences among the three models are small.

For all the other elements, the pollution of the interstellar medium is extremely dependent on the convective model:

- In the MLT17 case we would expect little oxygen depletion, and extremely large sodium and nitrogen enhancement. The sum $\mathrm{C}+\mathrm{N}+\mathrm{O}$ would be increased by $\sim 0.8$ dex, due to the effects of several TDUs. We would also probably expect strong s-process enrichment. The isotopic ratios of magnesium ${ }^{25} \mathrm{Mg} /{ }^{24} \mathrm{Mg}$ and ${ }^{26} \mathrm{Mg} /{ }^{24} \mathrm{Mg}$ would be slightly less than unity. These results are consistent with recent AGB models of the same metallicity presented by Fenner et al. (2004).

- In the MLT21 model we would expect a larger oxygen depletion and lower sodium enhancement. The increase of the sum $\mathrm{C}+\mathrm{N}+\mathrm{O}$ would be lower $(\sim 0.4 \mathrm{dex})$. The isotopic ratios of magnesium would both be around $\sim 3$.

- The FST model achieves the largest oxygen depletion, while the sodium content is slightly underabundant with respect to the solar value. The $\mathrm{C}+\mathrm{N}+\mathrm{O}$ abundance is conserved; the isotopic ratios of magnesium are similar to the MLT21 case.

In summary, we have seen that the stellar yields depend dramatically on the convection model. There are many other uncertainties in the results, but those are less critical.

We take as an example the sodium content of the ejecta: we saw that the results change significantly according to the efficiency of convection at the base of the outer convective zone. Figure 12 shows the variation with mass of the surface sodium abundance within the three models discussed so far (full, dotted, and dashed lines), plus a further FST model (dashed-dotted line) calculated by assuming the lower limit given in Angulo et al. (1999) for the reactions destroying sodium $\left({ }^{23} \mathrm{Na}(p, \gamma){ }^{24} \mathrm{Mg}\right.$ and $\left.{ }^{23} \mathrm{Na}(p, \alpha){ }^{20} \mathrm{Ne}\right)$ and the upper limit for reactions creating sodium $\left({ }^{22} \mathrm{Na}(p, \gamma)^{23} \mathrm{Mg}\right.$ and $\left.{ }^{22} \mathrm{Ne}(p, \gamma)^{23} \mathrm{Na}\right)$. We see that the influence of changing all the cross-sections involving sodium is far less than the role played by convection.

The problems of reaction rates and mass loss will be discussed in a forthcoming paper. 


\section{What implications are there for the self-enrichment scenario?}

Deep spectroscopic observations of giant and TO stars within globular clusters have shown star-to-star variations in surface chemical composition. Particularly, the observations of NGC 6752 by Gratton et al. (2001) showed the presence of an $\mathrm{O}-\mathrm{Na}$ anticorrelation in all stars observed, independently of their evolutionary stage, including those near the TO. Within the same cluster, strong hints of a $\mathrm{Mg}-\mathrm{Al}$ anticorrelation were also found. Similar results were obtained for other clusters (NGC 6397, M 30 and M 55: Carretta 2003; M 71: Ramirez \& Cohen 2002; M 5: Ramirez \& Cohen 2003). These results have also been recently confirmed by a deep analysis of the surface abundances of giant stars belonging to M 3 and M 13 by Sneden et al. (2004), where a clear C-O anticorrelation was found.

The aforementioned results indicate that the surface matter of these stars has been subject to nuclear processing though $\mathrm{CNO}, \mathrm{Na}-\mathrm{Ne}$ and $\mathrm{Mg}-\mathrm{Al}$ chains. Since these anomalies were observed also in TO stars, where the internal temperatures are too low to allow such nuclear reactions, it was suggested (Ventura et al. 2001; Ventura et al. 2002) that the stars displaying such surface chemical anomalies must have been contaminated by the ejecta expelled during the AGB evolution of an early generation of intermediate mass stars (Cottrell \& Da Costa 1981; D'Antona et al. 1983), or must have been formed by these ejecta (D'Antona et al. 2002).

Recently, this scenario has been questioned by Denissenkov \& Weiss (2001). Denissenkov \& Herwig (2003), based on the computations of a $5 M_{\odot}$ model with metallicity $Z=0.0001$, showed that a simultaneous depletion of oxygen and enhancement of sodium (as indicated by the anticorrelation observed) is made very difficult by sodium burning at high temperatures, those necessary to deplete oxygen; their analysis is independent of the amount of extra mixing assumed at the base of the convective envelope.

We postpone to a forthcoming paper the discussion of the role which IMS may play in the pollution of the interstellar medium within GCs, because a more complete analysis, covering the whole range of masses involved, and the most appropriate metallicities, rather than a single model, is required.

In any case, the present work clearly suggests that among all the uncertainties included in the input physics adopted to calculate the evolutionary sequences the treatment of convection has the greatest impact. AGB evolution is one of the few cases in stellar astrophysics where the results obtained are globally dependent on the convective model adopted, in terms of time scale, luminosity, temperature and nucleosynthesis.

Let us summarize again what we can say about some of the important elements for the self-enrichment scenario.

1. Lithium: the constant lithium content of the ejecta, independent of the convective model, is in good agreement with the observed lithium abundances of NGC 6397 TO stars (Bonifacio et al. 2002), which all show approximately the same population II standard value.

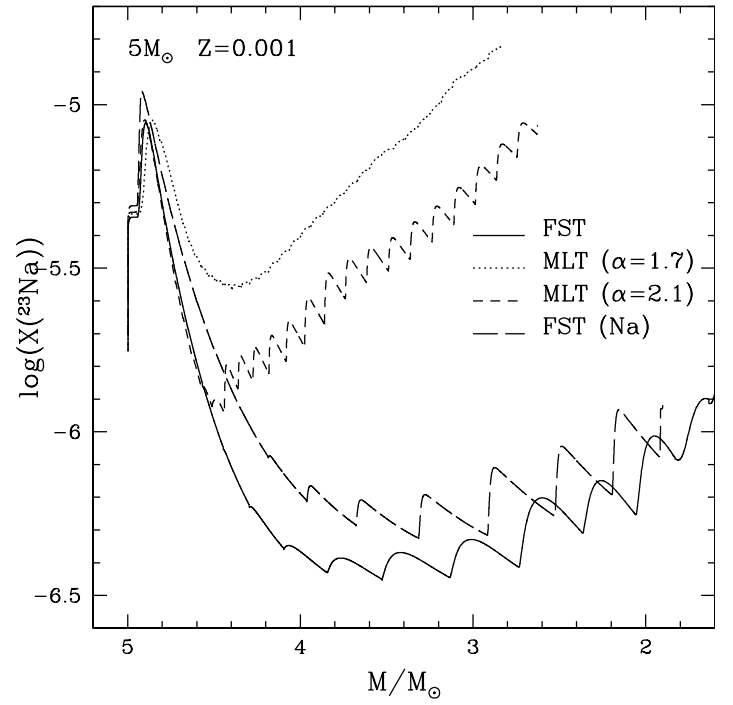

Fig. 12. The variation of the surface sodium abundance with mass within the three models precedently discussed, and a further FST model where sodium production is favoured chosing an appropriate nuclear network.

2. Helium: D'Antona et al. (2002) and D'Antona \& Caloi (2004) have shown that the high helium content of the ejecta, confirmed by the present calculations, plays a role in the morphology of the horizontal branches of GCs.

3. Sodium and Magnesium: from Fig. 12 we see that the MLT models can achieve sodium production quite efficiently, because of the TDU carrying fresh ${ }^{22} \mathrm{Ne}$ (later converted into sodium) to the surface; on the other hand, the FST model, living shorter because of a larger mass loss during the whole AGB evolution, ejects almost all the envelope mass well before the stage when TDU can operate significantly.

In terms of sodium-oxygen anticorrelation, if the adopted mass loss rate holds, only the MLT21 model seems to pollute the interstellar medium in the "right" direction, because apart from producing sodium efficiently it also depletes oxygen (see Table 1 and Fig. 11), though other problems arise, because in this case the polluted material would have magnetisium isotope ratios ${ }^{25} \mathrm{Mg} /{ }^{24} \mathrm{Mg}$ and ${ }^{26} \mathrm{Mg} /{ }^{24} \mathrm{Mg}$ around $\sim$, which disagrees with the measured magnesium isotopic ratios by Yong et al. (2003) for giant stars in NGC 6752. This problem is shared by the FST model.

4. $\mathrm{CNO}$ : the $\mathrm{C}+\mathrm{N}+\mathrm{O}$ content of the ejecta is almost unchanged with respect to the initial value in the FST models, which would be in agreement with the almost constant $\mathrm{C}+\mathrm{N}+\mathrm{O}$ value found for various GCs stars observed (M 92: Pilachowski et al. (1988); NGC 288 and NGC 362: Dickens et al. (1991); M 3 and M 13: Smith et al. (1996); M 4: Ivans et al. (1999)). The MLT models lead to a strong increase of the $\mathrm{C}+\mathrm{N}+\mathrm{O}$ abundance, due to the effects of several TDUs.

The self-enrichment hypothesis must be evaluated on the basis of a much deeper analysis, but it is clear that the uncertainties connected to convection can no longer be neglected in the computation of the ejecta of the various models. 


\section{Conclusions}

We examine the effects of changing the treatment of convection on the overall evolution of intermediate mass stars. We compare the stellar evolution models of initial mass $M=5 M_{\odot}$ calculated with the MLT treatment of the instability regions with those calculated with the FST model for turbulent convection. We show that while the evolutionary phases preceding TPs are scarcely affected (with the exception of RGB and early AGB effective temperatures), the AGB evolution is heavily influenced by the convective model adopted.

A higher convection efficiency at the bottom of the outer convective zone of AGBs leads to a shorter distance between the edge of the CNO-burning shell and the base of external convection; this, in turn, leads to an extra- luminosity (which, in the first TPs, is entirely due to ${ }^{3} \mathrm{He}$ burning) which triggers a faster growth of the luminosity itself, hence of mass loss.

From the physical point of view, the overall duration of the AGB evolution might be shorter by a factor of $\sim 3$ if the MLT treatment of convection is replaced by the FST model. Noticeable changes can be seen even if, still in the MLT framework, the free parameter $\alpha$ is changed. The maximum luminosity achieved by the FST is $\sim 70 \%$ larger, and the number of TPs (hence, of third dredge-up episodes) experienced by the FST model is considerably lower.

As for the chemical content of the ejecta, the only robust conclusion which can be drawn is that these stars pollute the interstellar medium with material which is extremely Heliumrich $\left(Y_{\text {ejecta }}=0.31\right.$ in all cases $)$ and with a lithium content which is only a factor of two lower than the standard population II value.

The extent of oxygen depletion, of sodium enrichment and of the total $\mathrm{C}+\mathrm{N}+\mathrm{O}$ abundance of the ejecta are strongly dependent on the convective model adopted.

Convection is one of the most important parameters in the AGB evolution. Any evaluation of the validity of the selfenrichment scenario for GCs must deal with the uncertainties connected to convection before deriving conclusions.

\section{References}

Angulo, C., et al. 1999, Nucl. Phys. A, 656, 3

Blöcker, T. 1995, A\&A, 297, 727

Blöcker, T., \& Schönberner, D. 1991, A\&A, 244, L43

Böhm-Vitense, E. 1958, Z. Astroph., 46, 108

Bonifacio, P., Pasquini, L., Spite, F., et al. 2002, A\&A, 390, 91

Cameron, A. G. V., \& Fowler, W. A. 1971, ApJ, 164, 111

Canuto, V. M., Goldman, I., \& Mazzitelli, I. 1996, ApJ, 473, 570

Canuto, V. M., \& Mazzitelli, I. 1991, ApJ, 370, 295

Carretta, E. 2003, Mem. S.A.It. Suppl., 3, 90
Caughlan, G. R., \& Fowler, W. A. 1988, Atomic Data Nucl. Tab., 40, 283

Cloutman, L., \& Eoll, J. G. 1976, ApJ, 206, 548

Cloutman, L., \& Whitaker, R. W. 1980, ApJ, 237, 900

Cottrell, P. L., \& Da Costa, G. S. 1981, ApJ, 245, 79

D’Antona, F., \& Caloi, V. 2004, ApJ, 611, 871

D'Antona, F., Caloi, V., Montalban, J., Ventura, P., \& Gratton, R. 2002, A\&A, 395, 69

D’Antona, F., Gratton, R., \& Chieffi, A. 1983, Mem. S.A.It., 54, 173

D’Antona, F., \& Mazzitelli, I. 1996, ApJ, 470, 1093

Deng, L., Bressan, A., \& Chiosi, C. 1996a, A\&A, 313, 145

Deng, L., Bressan, A., \& Chiosi, C. 1996b, A\&A, 313, 159

Denissenkov, P., \& Herwig, F. 2003, ApJ, 590, L99

Denissenkov, P., \& Weiss, A. 2001, ApJ, 559, L115

Dickens, R. J., Croke, B. F. W., Cannon, R. D., \& Bell, R. A. 1991, Nature, 351, 212

Fenner, Y., Campbell, S., Karakas, A. I., Lattanzio, J. C., \& Gibson, B. K. 2004, MNRAS, 353, 789

Freytag, B., Ludwig, H. G., \& Steffen, M. 1996, A\&A, 313, 497

Gratton, R., Bonifacio, P., Bragaglia, A., et al. 2001, A\&A, 369, 87

Grossman, S. A. 1996, MNRAS, 279, 305

Grossman, S. A., \& Narayan, R. 1993, ApJS, 89, 361

Hherwig, F. 2000, A\&A, 360, 952

Herwig, F. 2004, A\&A, 605, 425

Herwig, F., Blöcker, T., \& Schönberner, D. 1997, A\&A, 324, L81

Iben, I. J. 1975, ApJ, 196, 525

Iben, I. J. 1976, ApJ, 208, 165

Ivans, I. I., Sneden, C., Kraft, R. P., et al. 1999, AJ, 118, 1273

Mazzitelli, I., D’Antona, F., \& Ventura, P. 1999, A\&A, 348, 846

Pilachowski, C. A. 1988, ApJ, 326, L57

Ramirez, S., \& Cohen, J. G. 2002, AJ, 123, 3277

Ramirez, S., \& Cohen, J. G. 2003, AJ, 125, 224

Renzini, A., \& Voli, M. 1981, A\&A, 94, 175

Sackmann, J., \& Boothroyd, A. I. 1991, ApJ, 366, 529

Schwarzschild, M., \& Harm, R. 1965, ApJ, 142, 855

Schwarzschild, M., \& Harm, R. 1967, A\&A, 145, 486

Smith, G. H., Shetrone, M. D., Bell, R. A., Churchill, C. W., \& Briley, M. M. 1996, AJ, 112, 1511

Sneden, C., Kraft, R. P., Guhathakurta, P., Peterson, R. C., \& Fulbright, J. P. 2004, AJ, 127, 2162

Tuchman, Y., Glasner, A., \& Barkat, Z. 1983, ApJ, 268, 356

Ventura, P., \& Castellani, M. 2005, A\&A, 430, 1035

Ventura, P., D’Antona, F., Mazzitelli, I., \& Gratton, R. 2001, ApJ, 550, L65

Ventura, P., D’Antona, F., \& Mazzitelli, I. 2002, A\&A, 393, 215

Ventura, P., D’Antona, F., \& Mazzitelli, I. 2000, A\&A, 363, 605

Ventura, P., Zeppieri, A., D’Antona, F., \& Mazzitelli, I. 1998, A\&A, 334, 953

Vitense, E. 1953, Zs. Ap., 32, 135

Xiong, D. R. 1980, ChA, 4, 234

Xiong, D. R. 1985, A\&A, 150, 133

Xiong, D. R., Cheng, Q. L., \& Deng, L. 1997, ApJS, 108, 529

Yong, D., Grundahl, F., Nissen, P. E., \& Shetrone, M. D. 2003, A\&A, 402, 985 